\title{
Base subsets of symplectic Grassmannians
}

\author{
Mark Pankov
}

Received: 5 April 2006 / Accepted: 22 November 2006 /

Published online: 10 January 2007

(C) Springer Science + Business Media, LLC 2007

\begin{abstract}
Let $V$ and $V^{\prime}$ be $2 n$-dimensional vector spaces over fields $F$ and $F^{\prime}$. Let also $\Omega: V \times V \rightarrow F$ and $\Omega^{\prime}: V^{\prime} \times V^{\prime} \rightarrow F^{\prime}$ be non-degenerate symplectic forms. Denote by $\Pi$ and $\Pi^{\prime}$ the associated $(2 n-1)$-dimensional projective spaces. The sets of $k$ dimensional totally isotropic subspaces of $\Pi$ and $\Pi^{\prime}$ will be denoted by $\mathcal{G}_{k}$ and $\mathcal{G}_{k}^{\prime}$, respectively. Apartments of the associated buildings intersect $\mathcal{G}_{k}$ and $\mathcal{G}_{k}^{\prime}$ by so-called base subsets. We show that every mapping of $\mathcal{G}_{k}$ to $\mathcal{G}_{k}^{\prime}$ sending base subsets to base subsets is induced by a symplectic embedding of $\Pi$ to $\Pi^{\prime}$.
\end{abstract}

Keywords Tits building · Symplectic Grassmannians · Base subsets

\section{Introduction}

An incidence geometry of rank $n$ has the following ingredients: a set $\mathcal{G}$ whose elements are called subspaces, a symmetric incidence relation on $\mathcal{G}$, and a surjective dimension function

$$
\operatorname{dim}: \mathcal{G} \rightarrow\{0,1, \ldots, n-1\}
$$

such that the restriction of this function to every maximal flag is bijective (flags are sets of mutually incident subspaces).

A Tits building [12] is an incidence geometry together with a family of isomorphic subgeometries called apartments and satisfying a certain collection of axioms. One of these axioms says that for any two flags there is an apartment containing them.

M. Pankov $(\bowtie)$

Department of Mathematics and Information Technology, University of Warmia and Mazury,

Żolnierska 14A, 10-561 Olsztyn, Poland

e-mail: pankov@matman.uwm.edu.pl 
Let us consider an incidence geometry of rank $n$ whose set of subspaces is denoted by $\mathcal{G}$. For every $k \in\{0,1, \ldots, n-1\}$ we denote by $\mathcal{G}_{k}$ the Grassmannian consisting of all $k$-dimensional subspaces. If this geometry is a building then the intersection of $\mathcal{G}_{k}$ with an apartment is called the shadow of this apartment in $\mathcal{G}_{k}$ [12]. In the projective and symplectic cases the intersections of apartments with Grassmannians are known as base subsets [8-10].

Let $f$ be a bijective transformation of $\mathcal{G}_{k}$ preserving the class of the shadows of apartments. It is natural to ask: can $f$ be extended to an automorphism of the corresponding geometry? This problem was solved in [8] for buildings of type $A_{n}$, in this case $f$ is induced by a collineation of the associated projective space to itself or the dual projective space (the second possibility can be realized only for the case when $n=2 k+1$ ). A more general result can be found in [9].

In the present paper we show that the extension is possible for symplectic buildings.

Note that apartment preserving transformations of the chamber set (the set of maximal flags) of a spherical building are induced by automorphisms of the corresponding complex; this follows from the results given in [1].

\section{Symplectic geometry}

Let $V$ be a $2 n$-dimensional vector space over a field $F, n \geq 2$. Let also

$$
\Omega: V \times V \rightarrow F
$$

be a non-degenerate symplectic form. Denote by $\Pi=(P, \mathcal{L})$ the $(2 n-1)$-dimensional projective space associated with $V$ (points are 1-dimensional subspaces of $V$ and lines are defined by 2-dimensional subspaces).

We say that two points $p, q \in P$ are orthogonal and write $p \perp q$ if

$$
p=\langle x\rangle, \quad q=\langle y\rangle \quad \text { and } \quad \Omega(x, y)=0 .
$$

Similarly, two subspaces $S$ and $U$ of $\Pi$ will be called orthogonal $(S \perp U$ ) if $p \perp q$ for any $p \in S$ and $q \in U$. The orthogonal complement to a subspace $S$ (the maximal subspace orthogonal to $S$ ) will be denoted by $S^{\perp}$, if $S$ is $k$-dimensional then the dimension of $S^{\perp}$ is equal to $2 n-k-2$ (throughout the paper the dimension is always assumed to be projective).

A base $\left\{p_{1}, \ldots, p_{2 n}\right\}$ of $\Pi$ is said to be symplectic if for each $i \in\{1, \ldots, 2 n\}$ there exists unique $\sigma(i) \in\{1, \ldots, 2 n\}$ such that

$$
p_{i} \not \perp p_{\sigma(i)}
$$

( $p_{i}$ and $p_{\sigma(i)}$ are non-orthogonal).

A subspace $S$ of $\Pi$ is called totally isotropic if any two points of $S$ are orthogonal; in other words, $S \subset S^{\perp}$. The latter inclusion implies that the dimension of a totally isotropic subspace is not greater than $n-1$.

Now consider the incidence geometry of totally isotropic subspaces. For every symplectic base $B$ the subgeometry consisting of all totally isotropic subspaces spanned Springer 
by points of $B$ is the symplectic apartment associated with $B$. It is well-known that the incidence geometry of totally isotropic subspaces together with the family of all symplectic apartments is a building of type $C_{n}$.

For every $k \in\{0,1, \ldots, n-1\}$ we write $\mathcal{G}_{k}$ for the set of all $k$-dimensional totally isotropic subspaces, it is clear that $\mathcal{G}_{0}$ coincides with $P$. The set of all $k$-dimensional totally isotropic subspaces spanned by points of a symplectic base will be called the base subset of $\mathcal{G}_{k}$ associated with (defined by) this base.

Proposition 1. Every base subset of $\mathcal{G}_{k}$ consists of

$$
2^{k+1}\left(\begin{array}{c}
n \\
k+1
\end{array}\right)
$$

elements.

Proof: Let $B=\left\{p_{1}, \ldots, p_{2 n}\right\}$ be a symplectic base and $\mathcal{B}$ be the associated base subset of $\mathcal{G}_{k}$. By definition, $\mathcal{B}$ consists of all $k$-dimensional subspaces

$$
\overline{\left\{p_{i_{1}}, \ldots, p_{i_{k+1}}\right\}}
$$

such that

$$
\left\{i_{1}, \ldots, i_{k+1}\right\} \cap\left\{\sigma\left(i_{1}\right), \ldots, \sigma\left(i_{k+1}\right)\right\}=\emptyset .
$$

There are $2 n$ possibilities to choose $p_{i_{1}}$, then $p_{i_{2}}$ can be chosen in $2 n-2$ ways, and so on. Since the order of the points must not be taken into account, we obtain that $\mathcal{B}$ has precisely

$$
\frac{2 n \cdot(2 n-2) \ldots(2 n-2 k)}{(k+1) !}=2^{k+1}\left(\begin{array}{c}
n \\
k+1
\end{array}\right)
$$

elements.

Proposition 2. For any two k-dimensional totally isotropic subspaces there is a base subset of $\mathcal{G}_{k}$ containing them.

Proposition 2 can be obtained by an immediate verification or can be drawn from the fact that for any two flags there is an apartment containing them.

\section{Results}

From this moment we suppose that $V$ and $V^{\prime}$ are $2 n$-dimensional vector spaces over fields $F$ and $F^{\prime}$ (respectively), $n \geq 2$, and

$$
\Omega: V \times V \rightarrow F, \quad \Omega^{\prime}: V^{\prime} \times V^{\prime} \rightarrow F^{\prime}
$$


are non-degenerate symplectic forms. Let $\Pi=(P, \mathcal{L})$ and $\Pi^{\prime}=\left(P^{\prime}, \mathcal{L}^{\prime}\right)$ be the $(2 n-$ 1)-dimensional projective spaces associated with $V$ and $V^{\prime}$, respectively.

An injection $f: P \rightarrow P^{\prime}$ is called an embedding of $\Pi$ to $\Pi^{\prime}$ if it maps lines to subsets of lines and for any line $L^{\prime} \in \mathcal{L}^{\prime}$ there is at most one line $L \in \mathcal{L}$ such that $f(L) \subset L^{\prime}$. An embedding is said to be strong if it sends independent subsets to independent subsets. Every strong embedding of $\Pi$ to $\Pi^{\prime}$ is induced by a semilinear injection of $V$ to $V^{\prime}$ (with respect to a monomorphism of the underlying fields) preserving the linear independence [3-5].

Our projective spaces have the same dimension, and strong embeddings of $\Pi$ to $\Pi^{\prime}$ (if they exist) map bases to bases. An example given in [6] shows that strong embeddings of $\Pi$ to $\Pi^{\prime}$ cannot be characterized as mappings sending bases of $\Pi$ to bases of $\Pi^{\prime}$.

Theorem 1. If a mapping $f: P \rightarrow P^{\prime}$ transfers symplectic bases to symplectic bases then $f$ is a strong embedding of $\Pi$ to $\Pi^{\prime}$ and for any $p, q \in P$

$$
p \perp q \Longleftrightarrow f(p) \perp f(q) .
$$

Since a surjective embedding is a collineation, we get the following.

Corollary. Every surjection of $P$ to $P^{\prime}$ sending symplectic bases to symplectic bases is a collineation of $\Pi$ to $\Pi^{\prime}$ preserving the orthogonality relation.

In what follows embeddings and collineations sending symplectic bases to symplectic bases will be called symplectic.

For every $k \in\{0,1, \ldots, n-1\}$ we denote by $\mathcal{G}_{k}$ and $\mathcal{G}_{k}^{\prime}$ the sets of $k$-dimensional totally isotropic subspaces of $\Pi$ and $\Pi^{\prime}$, respectively.

Let $f: P \rightarrow P^{\prime}$ be a symplectic embedding of $\Pi$ to $\Pi^{\prime}$. For each $S \in \mathcal{G}_{k}$ the subspace spanned by $f(S)$ is an element of $\mathcal{G}_{k}^{\prime}$. The mapping

$$
\begin{aligned}
(f)_{k}: \mathcal{G}_{k} & \rightarrow \mathcal{G}_{k}^{\prime} \\
S & \rightarrow \overline{f(S)}
\end{aligned}
$$

is an injection sending base subsets to base subsets. If $f$ is a collineation then every $(f)_{k}$ is bijective. Conversely, an easy verification shows that if $(f)_{k}$ is bijective for certain $k$ then $f$ is a collineation.

Theorem 2. If a mapping of $\mathcal{G}_{k}$ to $\mathcal{G}_{k}^{\prime}(1 \leq k \leq n-1)$ transfers base subsets to base subsets then it is induced by a symplectic embedding of $\Pi$ to $\Pi^{\prime}$.

Corollary. Every surjection of $\mathcal{G}_{k}$ to $\mathcal{G}_{k}^{\prime}(1 \leq k \leq n-1)$ sending base subsets to base subsets is induced by a symplectic collineation of $\Pi$ to $\Pi^{\prime}$. 
For $k=n-1 \geq 2$ Theorem 2 was established in [10]. In the present paper it will be proved for the general case.

Our proof of Theorem 2 is based on elementary properties of so-called inexact subsets (Section 5). If $k=n-1$ then all maximal inexact subsets are of the same type. The case when $k<n-1$ is more complicated: there are two different types of maximal inexact subsets.

Two elements of $\mathcal{G}_{k}, k \geq 1$ are called adjacent if their intersection belongs to $\mathcal{G}_{k-1}$. We say that two elements of $\mathcal{G}_{k}$ are ortho-adjacent if they are orthogonal and adjacent; this is possible only if $k<n-1$. Using inexact subsets we characterize the adjacency and ortho-adjacency relations in terms of base subsets. This characterization shows that every mapping of $\mathcal{G}_{k}$ to $\mathcal{G}_{k}^{\prime}$ sending base subsets to base subsets is adjacency and ortho-adjacency preserving (Section 7); after that arguments in the spirit of [2] give the claim (Section 8).

\section{Proof of Theorem 1}

A line of $\Pi$ or $\Pi^{\prime}$ is said to be hyperbolic if it is not totally isotropic.

Lemma 1. Let $p_{1}, p_{2}, p \in P$ be distinct points such that the line $p_{1} p_{2}$ is hyperbolic and $p \in p_{1} p_{2}$. Then for any symplectic base $B$ containing $p_{1}, p_{2}$

$$
\left(B \backslash\left\{p_{i}\right\}\right) \cup\{p\} \quad i=1,2
$$

are symplectic bases.

Proof: Direct verification.

Lemma 2. Let $p_{1}, p_{2}, p \in P$ be distinct points. If there exists a symplectic base $B$ such that $p_{1}, p_{2} \in B$ and (1) are symplectic bases then the line $p_{1} p_{2}$ is hyperbolic and $p \in p_{1} p_{2}$.

Proof: Let $B=\left\{p_{1}, p_{2}, \ldots, p_{2 n}\right\}$ be such symplectic base. Since $\left(B \backslash\left\{p_{1}\right\}\right) \cup\{p\}$ is a symplectic base, $p \not \perp p_{\sigma(1)}$. Similarly, $p \not \perp p_{\sigma(2)}$. Thus there is no symplectic base containing $p, p_{\sigma(1)}, p_{\sigma(2)}$; this implies that $\sigma(1)=2$. Therefore, the line $p_{1} p_{2}$ is hyperbolic; moreover, it is the orthogonal complement to the subspace spanned by $p_{3}, \ldots, p_{2 n}$. It is easy to see that $p \perp p_{i}$ for every $i \geq 3$. Thus $p$ is a point on $p_{1} p_{2}$.

Let $f: P \rightarrow P^{\prime}$ be a mapping which sends symplectic bases of $\Pi$ to symplectic bases of $\Pi^{\prime}$. Since for any two points there is a symplectic base containing them, $f$ is injective. By Lemmas 1 and 2, $f$ transfers hyperbolic lines to subsets of hyperbolic lines; in particular,

$$
p \not \perp q \Longrightarrow f(p) \not \perp f(q) \text {. }
$$


We prove that

$$
p \perp q \Longrightarrow f(p) \perp f(q) \text {. }
$$

Let $p, q \in P$ be distinct orthogonal points and $B$ be a symplectic base containing them. There is a unique point $p^{\prime} \in B$ such that $p \not \perp p^{\prime}$. Then $f(p) \not \perp f\left(p^{\prime}\right)$ and all other points of $f(B)$ are orthogonal to $f(p)$; in particular, we get $f(p) \perp f(q)$.

Now we can show that $f$ maps totally isotropic lines to subsets of totally isotropic lines. Let $L$ be a totally isotropic line of $\Pi$ and $p_{1}, p_{2}$ be distinct points on this line. We can choose a symplectic base $\left\{p_{1}, p_{2}, \ldots, p_{2 n}\right\}$ such that $L$ is the orthogonal complement to the subspace spanned by $p_{1}, p_{2}, \ldots, p_{2 n-2}$. Then $f(L)$ is contained in the line $f\left(p_{1}\right) f\left(p_{2}\right)$ which is the orthogonal complement to the subspace spanned by $f\left(p_{1}\right), f\left(p_{2}\right), \ldots, f\left(p_{2 n-2}\right)$.

We have established that every line goes to a subset of a line. So we need to show that $f$ maps every base of $\Pi$ to a base of $\Pi^{\prime}$. We will use the following fact.

Fact 1. [7] If $g: P \rightarrow P^{\prime}$ is an injection transferring lines to subsets of lines then for every subset $X \subset P$

$$
g(\bar{X}) \subset \overline{g(X)}
$$

in particular, $\overline{g(X)}$ coincides with $\overline{g(\bar{X})}$.

Let $B$ be a base of $\Pi$. Then

$$
\overline{f(B)}=\overline{f(\bar{B})}=\overline{f(P)}
$$

The $f$-image of any symplectic base is a symplectic base, hence $\overline{f(P)}=P^{\prime}$ and $\Pi^{\prime}$ is spanned by $f(B)$. Since $f$ is injective, $f(B)$ is a base of $\Pi^{\prime}$.

\section{Inexact subsets}

Let $B=\left\{p_{1}, \ldots, p_{2 n}\right\}$ be a symplectic base of $\Pi$. Let also $1 \leq k \leq n-1$ and $\mathcal{B}$ be the base subset of $\mathcal{G}_{k}$ associated with $B$. It was noted in the proof of Proposition 1 that $\mathcal{B}$ consists of all $k$-dimensional subspaces

$$
\overline{\left\{p_{i_{1}}, \ldots, p_{i_{k+1}}\right\}}
$$

such that

$$
\left\{i_{1}, \ldots, i_{k+1}\right\} \cap\left\{\sigma\left(i_{1}\right), \ldots, \sigma\left(i_{k+1}\right)\right\}=\emptyset .
$$

If $k=n-1$ then every element of $\mathcal{B}$ contains precisely one of the points $p_{i}$ or $p_{\sigma(i)}$ for each $i$. 
We write $\mathcal{B}(+i)$ and $\mathcal{B}(-i)$ for the sets of all elements of $\mathcal{B}$ which contain $p_{i}$ or do not contain $p_{i}$, respectively. For any $i_{1}, \ldots, i_{s}$ and $j_{1}, \ldots, j_{u}$ belonging to $\{1, \ldots, 2 n\}$ we define

$\mathcal{B}\left(+i_{1}, \ldots,+i_{s},-j_{1}, \ldots,-j_{u}\right):=\mathcal{B}\left(+i_{1}\right) \cap \cdots \cap \mathcal{B}\left(+i_{s}\right) \cap \mathcal{B}\left(-j_{1}\right) \cap \cdots \cap \mathcal{B}\left(-j_{u}\right)$

The set of all elements of $\mathcal{B}$ incident with a subspace $S$ will be denoted by $\mathcal{B}(S)$ (this set may be empty). Then $\mathcal{B}(-i)$ coincides with $\mathcal{B}(S)$, where $S$ is the subspace spanned by $B \backslash\left\{p_{i}\right\}$. It is trivial that

$$
\mathcal{B}(+i)=\mathcal{B}(+i,-\sigma(i))
$$

and for the case when $k=n-1$ we have

$$
\mathcal{B}(-i)=\mathcal{B}(+\sigma(i))=\mathcal{B}(+\sigma(i),-i) .
$$

Let $\mathcal{R} \subset \mathcal{B}$. We say that $\mathcal{R}$ is exact if there is only one base subset of $\mathcal{G}_{k}$ containing $\mathcal{R}$; otherwise, $\mathcal{R}$ will be called inexact. If $\mathcal{R} \cap \mathcal{B}(+i)$ is not empty then we define $S_{i}(\mathcal{R})$ as the intersection of all subspaces belonging to $\mathcal{R}$ and containing $p_{i}$, and we define $S_{i}(\mathcal{R}):=\emptyset$ if the intersection of $\mathcal{R}$ and $\mathcal{B}(+i)$ is empty. If

$$
S_{i}(\mathcal{R})=p_{i}
$$

for all $i$ then $\mathcal{R}$ is exact; the converse fails.

Lemma 3. Let $\mathcal{R} \subset \mathcal{B}$. Suppose that there exist distinct $i, j$ such that

$$
p_{j} \in S_{i}(\mathcal{R}) \text { and } p_{\sigma(i)} \in S_{\sigma(j)}(\mathcal{R})
$$

Then $\mathcal{R}$ is inexact.

Proof: On the line $p_{i} p_{j}$ we choose a point $p_{i}^{\prime}$ different from $p_{i}$ and $p_{j}$. The line $p_{\sigma(i)} p_{\sigma(j)}$ contains a unique point orthogonal to $p_{i}^{\prime}$; we denote this point by $p_{\sigma(j)}^{\prime}$. Then

$$
\left(B \backslash\left\{p_{i}, p_{\sigma(j)}\right\}\right) \cup\left\{p_{i}^{\prime}, p_{\sigma(j)}^{\prime}\right\}
$$

is a symplectic base. The associated base subset of $\mathcal{G}_{k}$ contains $\mathcal{R}$ and we get the claim.

Proposition 3. The subset $\mathcal{B}(-i)$ is inexact; moreover, if $k<n-1$ then this is a maximal inexact subset. In the case when $k=n-1$, the inexact subset $\mathcal{B}(-i)$ is not maximal.

Proof: Let us take a point $p_{i}^{\prime}$ on the line $p_{i} p_{\sigma(i)}$ different from $p_{i}$ and $p_{\sigma(i)}$. Then

$$
\left(B \backslash\left\{p_{i}\right\}\right) \cup\left\{p_{i}^{\prime}\right\}
$$


is a symplectic base and the associated base subset of $\mathcal{G}_{k}$ contains $\mathcal{B}(-i)$. Hence this subset is inexact.

Let $k<n-1$. For any $j \neq i$ we can choose distinct

$$
i_{1}, \ldots, i_{k} \in\{1, \ldots, 2 n\} \backslash\{i, j, \sigma(i), \sigma(j)\}
$$

such that

$$
\left\{i_{1}, \ldots, i_{k}\right\} \cap\left\{\sigma\left(i_{1}\right), \ldots, \sigma\left(i_{k}\right)\right\}=\emptyset
$$

The subspaces spanned by

$$
p_{i_{1}}, \ldots, p_{i_{k}}, p_{j} \quad \text { and } \quad p_{\sigma\left(i_{1}\right)}, \ldots, p_{\sigma\left(i_{k}\right)}, p_{j}
$$

belong to $\mathcal{B}(-i)$. Since the intersection of these subspaces is $p_{j}$, we have

$$
S_{j}(\mathcal{B}(-i))=p_{j} \quad \text { if } \quad j \neq i
$$

Let $U$ be an arbitrarily taken element of

$$
\mathcal{B} \backslash \mathcal{B}(-i)=\mathcal{B}(+i)
$$

This subspace is spanned by $p_{i}$ and some $p_{i_{1}}, \ldots, p_{i_{k}}$. Since $p_{i}$ is the unique point of $U$ orthogonal to $p_{\sigma\left(i_{1}\right)}, \ldots, p_{\sigma\left(i_{k}\right)},(2)$ shows that the subset

$$
\mathcal{B}(-i) \cup\{U\}
$$

is exact. This implies that the inexact subset $\mathcal{B}(-i)$ is maximal.

Now let $k=n-1$. We take an arbitrary element $U \in \mathcal{B}(+i)$. There exists $j$ such that $p_{\sigma(j)}$ does not belong to $U$. Then $p_{j}$ is a point of the subspace

$$
S_{i}(\mathcal{B}(-i) \cup\{U\})=U
$$

Since $p_{\sigma(i)}$ belongs to every element of $\mathcal{B}(-i)$ and $p_{\sigma(j)}$ does not belong to $U$,

$$
S_{\sigma(j)}(\mathcal{B}(-i))=S_{\sigma(j)}(\mathcal{B}(-i) \cup\{U\})
$$

contains $p_{\sigma(i)}$. By Lemma 3, the subset (3) is inexact and the inexact subset $\mathcal{B}(-i)$ is not maximal.

Proposition 4. If $j \neq i, \sigma(i)$ then

$$
\mathcal{R}_{i j}:=\mathcal{B}(+i,+j) \cup \mathcal{B}(+\sigma(i),+\sigma(j)) \cup \mathcal{B}(-i,-\sigma(j))
$$

is a maximal inexact subset. 
We remark that

$$
\mathcal{R}_{i j}=\mathcal{B}(+i,+j) \cup \mathcal{B}(-i)
$$

if $k=n-1$.

\section{Proof: Since}

$$
S_{i}\left(\mathcal{R}_{i j}\right)=p_{i} p_{j} \quad \text { and } \quad S_{\sigma(j)}\left(\mathcal{R}_{i j}\right)=p_{\sigma(j)} p_{\sigma(i)},
$$

Lemma 3 shows that $\mathcal{R}_{i j}$ is inexact. We want to show that

$$
S_{l}\left(\mathcal{R}_{i j}\right)=p_{l} \quad \text { if } \quad l \neq i, \sigma(j)
$$

Let $l \neq i, j, \sigma(i), \sigma(j)$. If $k \geq 2$ then there exist

$$
i_{1}, \ldots, i_{k-2} \in\{1, \ldots, n\} \backslash\{i, j, \sigma(i), \sigma(j), l, \sigma(l)\}
$$

such that

$$
\left\{i_{1}, \ldots, i_{k}\right\} \cap\left\{\sigma\left(i_{1}\right), \ldots, \sigma\left(i_{k}\right)\right\}=\emptyset
$$

the subspaces spanned by

$$
p_{i_{1}}, \ldots, p_{i_{k-2}}, p_{l}, p_{i}, p_{j} \quad \text { and } \quad p_{\sigma\left(i_{1}\right)}, \ldots, p_{\sigma\left(i_{k-2}\right)}, p_{l}, p_{\sigma(i)}, p_{\sigma(j)}
$$

are elements of $\mathcal{R}_{i j}$ intersecting in the point $p_{l}$. If $k=1$ then the lines $p_{l} p_{\sigma(i)}$ and $p_{l} p_{j}$ are as required.

Now we choose distinct

$$
i_{1}, \ldots, i_{k-1} \in\{1, \ldots, n\} \backslash\{i, j, \sigma(i), \sigma(j)\}
$$

such that

$$
\left\{i_{1}, \ldots, i_{k-1}\right\} \cap\left\{\sigma\left(i_{1}\right), \ldots, \sigma\left(i_{k-1}\right)\right\}=\emptyset
$$

and consider the subspace spanned by

$$
p_{i_{1}}, \ldots, p_{i_{k-2}}, p_{j}, p_{\sigma(i)} \text {. }
$$

This subspace intersects the subspaces spanned by

$$
p_{i_{1}}, \ldots, p_{i_{k-1}}, p_{j}, p_{i} \quad \text { and } \quad p_{i_{1}}, \ldots, p_{i_{k-1}}, p_{\sigma(i)}, p_{\sigma(j)}
$$

precisely in the points $p_{j}$ and $p_{\sigma(i)}$, respectively. Since all these subspaces are elements of $\mathcal{R}_{i j}$, we get (4) for $l=j, \sigma(i)$. 
A direct verification shows that

$$
\mathcal{B} \backslash \mathcal{R}_{i j}=\mathcal{B}(+i,-j) \cup \mathcal{B}(+\sigma(j),-\sigma(i))
$$

Thus for every $U \in \mathcal{B} \backslash \mathcal{R}_{i j}$ one of the following possibilities is realized:

(1) $U \in \mathcal{B}(+i,-j)$ intersects $S_{i}\left(\mathcal{R}_{i j}\right)=p_{i} p_{j}$ by $p_{i}$,

(2) $U \in \mathcal{B}(+\sigma(j),-\sigma(i))$ intersects $S_{\sigma(j)}\left(\mathcal{R}_{i j}\right)=p_{\sigma(j)} p_{\sigma(i)}$ by $p_{\sigma(j)}$.

Since $p_{\sigma(j)}$ is the unique point of the line $p_{\sigma(j)} p_{\sigma(i)}$ orthogonal to $p_{i}$ and $p_{i}$ is the unique point on $p_{i} p_{j}$ orthogonal to $p_{\sigma(j)}$, the subset

$$
\mathcal{R}_{i j} \cup\{U\}
$$

is exact for each $U$ belonging to $\mathcal{B} \backslash \mathcal{R}_{i j}$. Thus the inexact subset $\mathcal{R}_{i j}$ is maximal.

The maximal inexact subsets considered in Propositions 3 and 4 will be called of first and second type, respectively.

Proposition 5. Every maximal inexact subset is of first or second type. In particular, if $k=n-1$ then each maximal inexact subset is of second type.

Proof: Let $\mathcal{R}$ be a maximal inexect subset of $\mathcal{B}$, and let $B^{\prime}$ be another symplectic base of $\Pi$ such that the associated base subset of $\mathcal{G}_{k}$ contains $\mathcal{R}$. If certain $S_{i}(\mathcal{R})$ is empty then $\mathcal{R} \subset \mathcal{B}(-i)$. In the case when $k=n-1$, this is impossible (the inexact subset $\mathcal{B}(-i)$ is not maximal). If $k<n-1$ then the inverse inclusion holds (since our inexact subset is maximal).

Now suppose that each $S_{i}(\mathcal{R})$ is not empty. Denote by $I$ the set of all $i$ such that the dimension of $S_{i}(\mathcal{R})$ is non-zero. Since $\mathcal{R}$ is inexact, $I$ is non-empty. Suppose that for certain $l \in I$ the subspace $S_{l}(\mathcal{R})$ is spanned by $p_{l}, p_{j_{1}}, \ldots, p_{j_{u}}$ and

$$
M_{1}:=S_{\sigma\left(j_{1}\right)}(\mathcal{R}), \ldots, M_{u}:=S_{\sigma\left(j_{u}\right)}(\mathcal{R})
$$

do not contain $p_{\sigma(l)}$. Then $p_{l}$ belongs to $M_{1}^{\perp}, \ldots, M_{u}^{\perp}$; on the other hand,

$$
p_{j_{1}} \notin M_{1}^{\perp}, \ldots, p_{j_{u}} \notin M_{u}^{\perp}
$$

and we have

$$
M_{1}^{\perp} \cap \cdots \cap M_{u}^{\perp} \cap S_{l}(\mathcal{R})=p_{l} .
$$

Since $S_{1}(\mathcal{R}), \ldots, S_{2 n}(\mathcal{R})$ and their orthogonal complements are spanned by points of the base $B^{\prime}$, the point $p_{l}$ belongs to $B^{\prime}$. The fact that $B \neq B^{\prime}$ implies the existence of $i \in I$ and $j \neq i, \sigma(i)$ such that

$$
p_{j} \in S_{i}(\mathcal{R}) \text { and } p_{\sigma(i)} \in S_{\sigma(j)}(\mathcal{R}) \text {. }
$$

Then $\mathcal{R}=\mathcal{R}_{i j}$. 
Maximal inexact subsets of the same type have the same cardinality. These cardinalities will be denoted by $c_{1}(k)$ and $c_{2}(k)$, respectively. An immediate verification shows that each of the following possibilities

$$
c_{1}(k)=c_{2}(k), c_{1}(k)<c_{2}(k), c_{1}(k)>c_{2}(k)
$$

is realized for suitable $k$.

\section{Complement subsets}

Let $\mathcal{B}$ be as in the previous section. We say that $\mathcal{R} \subset \mathcal{B}$ is a complement subset if $\mathcal{B} \backslash \mathcal{R}$ is a maximal inexact subset. A complement subset is said to be of first or second type if the corresponding maximal inexact subset is of first or second type, respectively. The complement subsets for the maximal inexact subsets from Propositions 3 and 4 are

$$
\mathcal{B}(+i) \quad \text { and } \quad \mathcal{B}(+i,-j) \cup \mathcal{B}(+\sigma(j),-\sigma(i))
$$

If $k=n-1$ then the second subset coincides with

$$
\mathcal{B}(+i,+\sigma(j))=\mathcal{B}(+i,+\sigma(j),-j,-\sigma(i)) .
$$

In the case when $k=n-1=1$, a complement subset has one element only.

Lemma 4. Let $k=n-1 \geq 2$. Then $S, U \in \mathcal{B}$ are adjacent if and only if there are precisely $\left(\begin{array}{c}n-1 \\ 2\end{array}\right)$ distinct complement subsets of $\mathcal{B}$ containing both $S$ and $U$.

Proof: Denote by $m$ the dimension of $S \cap U$. The complement subset $\mathcal{B}(+i,+j)$ contains our subspaces if and only if $p_{i}, p_{j}$ belong to $S \cap U$. Thus there are precisely $\left(\begin{array}{c}m+1 \\ 2\end{array}\right)$ distinct complement subsets of $\mathcal{B}$ containing $S$ and $U$.

Lemma 5. Let $k<n-1$ and $\mathcal{R}$ be a complement subset of $\mathcal{B}$. If $\mathcal{R}$ is of first type then there are precisely $4 n-3$ distinct complement subsets of $\mathcal{B}$ which do not intersect $\mathcal{R}$. If $\mathcal{R}$ is of second type then there are precisely 4 distinct complement subsets of $\mathcal{B}$ which do not intersect $\mathcal{R}$.

To prove Lemma 11 we use the following.

Lemma 6. Let $k<n-1$ and $i, i^{\prime}, j, j^{\prime}$ be elements of $\{1, \ldots, 2 n\}$ such that $i \neq j$ and $i^{\prime} \neq j^{\prime}$. If the intersection of

$$
\mathcal{B}(+i,-j) \quad \text { and } \quad \mathcal{B}\left(+i^{\prime},-j^{\prime}\right)
$$

is empty then one of the following possibilities is realized: $i^{\prime}=\sigma(i), i^{\prime}=j, j^{\prime}=i$. 
Proof: Direct verification.

Proof of Lemma 5. Let us fix $l \in\{1, \ldots, 2 n\}$ and consider the complement subset $\mathcal{B}(+l)$. If $\mathcal{B}(+i)$ is disjoint with $\mathcal{B}(+l)$ then $i=\sigma(l)$. If for some $i, j \in\{1, \ldots, 2 n\}$ the complement subset

$$
\mathcal{B}(+i,-j) \cup \mathcal{B}(+\sigma(j),-\sigma(i))
$$

does not intersect $\mathcal{B}(+l)$ then one of the following possibilities is realized:

(1) $i=\sigma(l)$, the condition $j \neq i, \sigma(i)$ shows that there are precisely $2 n-2$ possibilities for $j$;

(2) $j=l$ and there are precisely $2 n-2$ possibilities for $i$ (since $i \neq j, \sigma(j)$ ).

Now fix $i, j \in\{1, \ldots, 2 n\}$ such that $j \neq i, \sigma(i)$ and consider the associated complement subset

$$
\mathcal{B}(+i,-j) \cup \mathcal{B}(+\sigma(j),-\sigma(i)) \text {. }
$$

There are only two complement subsets of the first type disjoint with (5):

$$
\mathcal{B}(+\sigma(i)) \quad \text { and } \quad \mathcal{B}(+j) .
$$

If

$$
\mathcal{B}\left(+i^{\prime},-j^{\prime}\right) \cup \mathcal{B}\left(+\sigma\left(j^{\prime}\right),-\sigma\left(i^{\prime}\right)\right)
$$

does not intersect (5) then one of the following two possibilities is realized:

$$
i^{\prime}=j, j^{\prime}=i \quad \text { or } \quad i^{\prime}=\sigma(i), j^{\prime}=\sigma(j)
$$

(see Lemma 12).

\section{Main lemma}

Let $f: \mathcal{G}_{k} \rightarrow \mathcal{G}_{k}^{\prime}(1 \leq k \leq n-1)$ be a mapping which sends base subsets to base subsets. Since for any two elements of $\mathcal{G}_{k}$ there exists a base subset containing them (Proposition 2) and the restriction of $f$ to every base subset of $\mathcal{G}_{k}$ is a bijection to a base subset of $\mathcal{G}_{k}^{\prime}$, the mapping $f$ is injective.

Throughout the section we suppose that $n \geq 3$. In this section the following statement will be proved.

Lemma 7 (Main Lemma). Let $S, U \in \mathcal{G}_{k}$. Then $S$ and $U$ are adjacent if and only if $f(S)$ and $f(U)$ are adjacent. Moreover, for the case when $k<n-1$, the subspaces $S$ and $U$ are ortho-adjacent if and only if the same holds for $f(S)$ and $f(U)$. 
Let $\mathcal{B}$ be a base subset of $\mathcal{G}_{k}$ containing $S$ and $U$. Then $\mathcal{B}^{\prime}:=f(\mathcal{B})$ is a base subset of $\mathcal{G}_{k}\left(\Omega^{\prime}\right)$ and the restriction $\left.f\right|_{\mathcal{B}}$ is a bijection to $\mathcal{B}^{\prime}$.

Lemma 8. A subset $\mathcal{R} \subset \mathcal{B}$ is inexact if and only if $f(\mathcal{R})$ is inexact; moreover, $\mathcal{R}$ is a maximal inexact subset if and only if the same holds for $f(\mathcal{R})$.

Proof: If $\mathcal{R}$ is inexact then there are two distinct base subsets of $\mathcal{G}_{k}$ containing $\mathcal{R}$ and their $f$-images are distinct base subsets of $\mathcal{G}_{k}^{\prime}$ containing $f(\mathcal{R})$, hence $f(\mathcal{R})$ is inexact. The base subsets $\mathcal{B}$ and $\mathcal{B}^{\prime}$ have the same number of inexact subsets and the first part of our statement is proved. Since $\mathcal{B}$ and $\mathcal{B}^{\prime}$ have the same number of maximal inexact subsets, every maximal inexact subset of $\mathcal{B}^{\prime}$ is the image of a maximal inexact subset of $\mathcal{B}$.

Lemma 9. $\mathcal{R} \subset \mathcal{B}$ is a complement subset if and only if $f(\mathcal{R})$ is a complement subset of $\mathcal{B}^{\prime}$.

Proof: This is a simple consequence of the previous lemma.

If $k=n-1$ then Main Lemma (Lemma 7) can be drawn directly from Lemmas 10 and 15. In [10] this statement was proved in a more complicated way.

Lemma 10. If $k<n-1$ then the mapping $\left.f\right|_{\mathcal{B}}$ together with the inverse mapping preserve types of maximal inexact and complement subsets.

Proof: This statement is trivial if $c_{1}(k) \neq c_{2}(k)$. In the general case it follows from Lemma 5.

We write $\mathcal{X}_{i}$ and $\mathcal{X}_{i}^{\prime}$ for the sets of all $i$-dimensional subspaces spanned by points of the symplectic bases associated with $\mathcal{B}$ and $\mathcal{B}^{\prime}$, respectively. We denote by $\mathcal{B}(N)$ and $\mathcal{B}^{\prime}\left(N^{\prime}\right)$ the sets of all elements of $\mathcal{B}$ and $\mathcal{B}^{\prime}$ incident with subspaces $N$ and $N^{\prime}$, respectively.

Lemma 11. Let $k<n-1$. There exists a bijection $g: \mathcal{X}_{k+1} \rightarrow \mathcal{X}_{k+1}^{\prime}$ such that

$$
f(\mathcal{B}(N))=\mathcal{B}^{\prime}(g(N))
$$

for every $N \in \mathcal{X}_{k+1}$.

Proof: Lemma 10 guarantees that $\left.f\right|_{\mathcal{B}}$ and the inverse mapping send maximal inexact subsets of first type to maximal inexact subsets of first type. This implies the existence of a bijection $h: \mathcal{X}_{2 n-2} \rightarrow \mathcal{X}_{2 n-2}^{\prime}$ such that

$$
f(\mathcal{B}(M))=\mathcal{B}^{\prime}(h(M))
$$


for all $M \in \mathcal{X}_{2 n-2}$. Each $N \in \mathcal{X}_{k+1}$ can be presented as the intersection of

$$
M_{1}, \ldots, M_{2 n-k-2} \in \mathcal{X}_{2 n-2} \text {. }
$$

Then

$$
g(N):=\bigcap_{i=1}^{2 n-k-2} h\left(M_{i}\right)
$$

is as required.

Now we prove Lemma 7 for $k<n-1$. Two subspaces $S, U \in \mathcal{B}$ are adjacent if and only if they belong to $\mathcal{B}(T)$ for certain $T \in \mathcal{X}_{k+1}$; moreover, $S$ and $U$ are orthoadjacent if and only if $\mathcal{B}(T)$ consists of $k+2$ elements (in other words, $T$ is totally isotropic). The required statement follows from Lemma 11.

\section{Proof of Theorem 2 for $n \geq 3$}

Let $M, N$ be a pair of incident subspaces of $\Pi \operatorname{such}$ that $\operatorname{dim} M<k<\operatorname{dim} N$. We denote by $[M, N]_{k}$ the set of $k$-dimensional subspaces of $\Pi$ incident with both $M$ and $N$; in the case when $M=\emptyset$ or $N=P$, we write $(N]_{k}$ or $[M)_{k}$, respectively.

We say that $\mathcal{X} \subset \mathcal{G}_{k}$ is an $A$-subset if any two distinct elements of $\mathcal{X}$ are adjacent.

Example 1. If $k<n-1$ and $N$ is an element of $\mathcal{G}_{k+1}$ then $(N]_{k}$ is a maximal $A$-subset of $\mathcal{G}_{k}$. Subsets of such type will be called tops. Any two distinct elements of a top are ortho-adjacent.

Example 2. If $M$ belongs to $\mathcal{G}_{k-1}$ then

$$
\left[M, M^{\perp}\right]_{k}=[M)_{k} \cap \mathcal{G}_{k}
$$

is a maximal $A$-subset of $\mathcal{G}_{k}$. Such maximal $A$-subsets are known as stars, they contain non-orthogonal elements.

Fact $2([2,11])$. Each $A$-subset is contained in a maximal $A$-subset. Every maximal $A$-subset of $\mathcal{G}_{n-1}$ is a star. If $k<n-1$ then every maximal $A$-subset of $\mathcal{G}_{k}$ is a top or a star.

Let $n \geq 3$ and $f$ be as in the previous section. The first part of Lemma 7 says that $f$ transfers $A$-subsets to $A$-subsets. The second part of Lemma 7 guarantees that stars go to subsets of stars. In other words, for any $M \in \mathcal{G}_{k-1}$ there exists $M^{\prime} \in \mathcal{G}_{k-1}^{\prime}$ such that

$$
f\left(\left[M, M^{\perp}\right]_{k}\right) \subset\left[M^{\prime}, M^{\prime \perp}\right]_{k} .
$$


Suppose that

$$
f\left(\left[M, M^{\perp}\right]_{k}\right) \subset\left[M^{\prime \prime}, M^{\prime \prime}\right]_{k}
$$

for $M^{\prime \prime} \in \mathcal{G}_{k-1}^{\prime}$ other than $M^{\prime}$. Then $f\left(\left[M, M^{\perp}\right]_{k}\right)$ is contained in the intersection of $\left[M^{\prime}, M^{\prime \perp}\right]_{k}$ and $\left[M^{\prime \prime}, M^{\prime \prime}\right]_{k}$. This intersection is not empty only if $M^{\prime}=M^{\prime \prime}$ or $M^{\prime}$ and $M^{\prime \prime}$ are ortho-adjacent; but in the second case our intersection consists of one element only. Thus there is unique $M^{\prime} \in \mathcal{G}_{k-1}^{\prime}$ satisfying (6). We have established the existence of a mapping

$$
g: \mathcal{G}_{k-1} \rightarrow \mathcal{G}_{k-1}^{\prime}
$$

such that

$$
f\left(\left[M, M^{\perp}\right]_{k}\right) \subset\left[g(M), g(M)^{\perp}\right]_{k}
$$

for every $M \in \mathcal{G}_{k-1}$. It is easy to see that

$$
g\left((N]_{k-1}\right) \subset(f(N)]_{k-1} \quad \forall N \in \mathcal{G}_{k} .
$$

Now we show that $g$ sends base subsets to base subsets.

Proof: Let $\mathcal{B}_{k-1}$ be a base subset of $\mathcal{G}_{k-1}$ and $B$ be the associated symplectic base. This base defines a base subset $\mathcal{B} \subset \mathcal{G}_{k}$. Now let $B^{\prime}$ be the symplectic base associated with the base subset $\mathcal{B}^{\prime}:=f(\mathcal{B})$ and $\mathcal{B}_{k-1}^{\prime}$ be the base subset of $\mathcal{G}_{k-1}^{\prime}$ defined by $B^{\prime}$. If $S \in \mathcal{B}_{k-1}$ then we take $U_{1}, U_{2} \in \mathcal{B}$ such that $S=U_{1} \cap U_{2}$, and (7) shows that

$$
g(S)=f\left(U_{1}\right) \cap f\left(U_{2}\right) \in \mathcal{B}_{k-1}^{\prime} .
$$

Thus $g\left(\mathcal{B}_{k-1}\right)$ is contained in $\mathcal{B}_{k-1}^{\prime}$. Suppose that $g\left(\mathcal{B}_{k-1}\right)$ is a proper subset of $\mathcal{B}_{k-1}^{\prime}$. Then $g(S)=g(U)$ for some $S, U \in \mathcal{B}_{k-1}$. The $f$-image of

$$
\mathcal{B}(S)=\mathcal{B} \cap\left[S, S^{\perp}\right]_{k}
$$

is contained in

$$
\mathcal{B}^{\prime}(g(S))=\mathcal{B}^{\prime} \cap\left[g(S), g(S)^{\perp}\right]_{k}
$$

Since these sets have the same cardinality,

$$
f(\mathcal{B}(S))=\mathcal{B}^{\prime}(g(S))
$$

Similarly,

$$
f(\mathcal{B}(U))=\mathcal{B}^{\prime}(g(U)) .
$$


The equality $f(\mathcal{B}(S))=f(\mathcal{B}(U))$ contradicts the injectivity of $f$. Hence $g\left(\mathcal{B}_{k-1}\right)$ coincides with $\mathcal{B}_{k-1}^{\prime}$.

If $k=1$ then the mapping $g: P \rightarrow P^{\prime}$ sends symplectic bases to symplectic bases. By Theorem 1, $g$ is a symplectic embedding of $\Pi$ to $\Pi^{\prime}$, and we have $f=(g)_{1}$.

Now suppose that $k>1$ and $g$ is induced by a symplectic embedding $h$ of $\Pi$ to $\Pi^{\prime}$. Let us consider an arbitrary element $S \in \mathcal{G}_{k}$ and take ortho-adjacent $M, N \in \mathcal{G}_{k-1}$ such that $S=\overline{M \cup N}$. Then

$$
\{S\}=\left[M, M^{\perp}\right]_{k} \cap\left[N, N^{\perp}\right]_{k}
$$

and $f(S)$ belongs to the intersection of $\left[g(M), g(M)^{\perp}\right]_{k}$ and $\left[g(N), g(N)^{\perp}\right]_{k}$. Since

$$
g(M)=\overline{h(M)} \quad \text { and } \quad g(N)=\overline{h(N)}
$$

are ortho-adjacent, the intersection of $\left[g(M), g(M)^{\perp}\right]_{k}$ and $\left[g(N), g(N)^{\perp}\right]_{k}$ consists of one element and we have

$$
f(S)=\overline{\overline{h(M)} \cup \overline{h(N)}}=\overline{h(S)} .
$$

This means that $f$ is induced by $h$. Therefore, Theorem 2 can be proved by induction.

\section{Proof of Theorem 2 for $n=2$}

Let $k=n-1=1$. In this case, a base subset consists of 4 elements.

For $S, U \in \mathcal{G}_{k}$ we denote by $\mathcal{X}(S, U)$ the set of all $M \in \mathcal{G}_{k} \backslash\{S, U\}$ such that there is a base subset containing $S, U, M$.

Since $k=1$, two distinct elements of $\mathcal{G}_{k}$ are adjacent or non-intersecting.

Lemma 12. Two distinct $S, U \in \mathcal{G}_{k}$ are non-intersecting if and only iffor any distinct $M, N \in \mathcal{X}(S, U)$

$$
\{S, U, M, N\}
$$

is a base subset.

Proof: Direct verification.

This is a partial case of a more general property of generalized polygons [13].

Let $f$ be as in Sections 7 and 8. Since

$$
f(\mathcal{X}(S, U)) \subset \mathcal{X}(f(S), f(U)),
$$

Lemma 12 shows that $f$ maps adjacent elements of $\mathcal{G}_{k}$ to adjacent elements of $\mathcal{G}_{k}^{\prime}$. Then stars go to subsets of stars and $f$ induces a mapping $g: P \rightarrow P^{\prime}$. As above, we establish that $g$ sends bases of $\Pi$ to bases of $\Pi^{\prime}$ and Theorem 1 gives the claim. 
Acknowledgements The author thanks one of the referees for a long list of valuable remarks and a shorter proof of Proposition 1.

\section{References}

1. P. Abramenko and H. Van Maldeghem, "On opposition in spherical buildings and twin buildings," Ann. Combinatorics 4 (2000), 125-137.

2. W.L. Chow, "On the geometry of algebraic homogeneous spaces," Ann. Math. 50 (1949), 32-67.

3. C.A. Faure and A. Frölicher, "Morphisms of projective geometries and semilinear maps," Geom. Dedicata 53 (1994), 237-262.

4. C.A. Faure, "An elementary proof of the fundamental theorem of projective geometry," Geom. Dedicata 90 (2002) 145-151.

5. H. Havlicek, "A generalization of Brauner's theorem on linear mappings," Mitt. Math. Sem. Univ. Giessen 215 (1994), 27-41.

6. W.-1. Huang and A. Kreuzer, "Basis preserving maps of linear spaces," Arch. Math. (Basel) 64(6) (1995), 530-533.

7. A. Kreuzer, "On the definition of isomorphisms of linear spaces," Geom. Dedicata 61 (1996), 279-283.

8. M. Pankov, "Transformations of Grassmannians and automorphisms of classical groups," J. Geom. 75 (2002), 132-150.

9. M. Pankov, "A characterization of geometrical mappings of Grassmann spaces," Results Math. 45 (2004), 319-327.

10. M. Pankov, "Mappings of the sets of invariant subspaces of null systems," Beiträge zur Algebra Geom. 45 (2004), 389-399.

11. M. Pankov, K. Prażmovski, and M. Żynel, "Geometry of polar Grassmann spaces," Demonstratio Math. 39 (2006), 625-637.

12. J. Tits, Buildings of Spherical Type and Finite BN-Pairs, Lecture Notes in Mathematics 386, Springer, Berlin, 1974.

13. H. Van Maldeghem, Private communication. 